\title{
ENHANCED RADAR SCATTERING FROM WATER INCLUSIONS IN ICE
}

\author{
By J.L. BAMBER
}

(Scott Polar Research Institute, University of Cambridge, Cambridge CB2 1ER, England)

ABSTRACT. It is believed that water-filled cavities on the scale of decimetres are present in a number of glaciers in Spitsbergen. The evidence for this hypothesis comes from airborne radio echo-sounding records collected by the Scott Polar Research Institute in 1983. The size of these cavities prevents the use of Rayleigh scattering theory and requires a more general analysis. Mie scattering theory is used to investigate the scattered power providing some unexpected results, the most significant of which is that for water bodies greater than approximately $0.25 \mathrm{~m}$ in radius the returned power from the volume illuminated is greater than that from a perfectly reflecting plane boundary.

\section{INTRODUCTION}

Recent radio echo-sounding (RES) studies carried out at a sounding frequency of $60 \mathrm{MHz}$ (Bamber, unpublished) and results of bore-hole investigations (Hodge, 1976; Schommer, 1977) suggest the existence of englacial inhomogeneities on the scale of decimetres. Previous attempts to model the scattered power from inclusions in natural ice have generally assumed that they are very much smaller than the sounding wavelengths which typically lie in the range $0.4-3 \mathrm{~m}$ (in ice). Consequently, Rayleigh scattering theory (which requires that $2 \pi a / \lambda \ll 1$ ) is not applicable and to determine the back-scattered power from cavities of this size a more general analysis is necessary.

Mie's theory of scattering of light, by particles of arbitrary size, provides an approach in which the only assumptions required are that scatterers be spherical and act as independent sources (i.e. there is no interference between the power radiated by different scatterers), so that the total scattered power is the sum of that from each target. The latter restriction is satisfied if the distance between each source is greater than approximately three times the radius of the scatterer: it is not a restrictive criterion. The former is less likely to be obeyed but the results are not significantly affected (van de Hulst, 1957).

Mie's theory is used to calculate, by numerical analysis, the back-scattering cross-section $\sigma$ of a body of arbitrary size, and hence the returned power using the radar equation

$$
P_{\mathrm{r}}=P_{\mathrm{t}} \frac{G^{2} \lambda^{2}}{(4 \pi)^{3} r^{4}} \sigma,
$$

where $P_{\mathrm{r}}$ and $P_{\mathrm{t}}$ are the received and transmitted power respectively, $G$ is the forward antenna gain, $\lambda$ is the radar wavelength in the sounding medium, and $r$ is the distance between the antenna and the scattering centre. The analysis follows that of van de Hulst (1957).

For backward scattering $\left(\theta=180^{\circ}\right)$, the scattering amplitude for arbitrarily polarized radiation is

$$
S_{1}(180)=-\sum_{n=1}^{\infty}(n+1 / 2)(-1)^{n}\left(a_{n}-b_{n}\right)
$$

where $a_{n}$ and $b_{n}$ are the Mie coefficients

$$
\begin{aligned}
a_{n} & =\frac{\psi_{n}^{\prime}(y) \Psi_{n}(x)-m^{*} \Psi_{n}(y) \Psi_{n}^{\prime}(x)}{\psi_{n}^{\prime}(y) \zeta_{n}(x)-m^{*} \Psi_{n}(y) \zeta_{n}^{\prime}(x)}, \\
b_{n} & =\frac{m^{*} \psi_{n}^{\prime}(y) \Psi_{n}(x)-\Psi_{n}(y) \psi_{n}^{\prime}(x)}{m^{*} \psi_{n}^{\prime}(y) \zeta_{n}(x)-\psi_{n}(y) \zeta_{n}^{\prime}(x)},
\end{aligned}
$$

$x=2 \pi a / \lambda=K a$ and $y=m^{*} k a$, where $m^{*}$ is the complex refractive index

$$
m^{* 2}=\varepsilon^{\prime}-i \varepsilon^{\prime \prime} \quad m^{*}=n^{\prime}-i n^{\prime \prime} .
$$

For $\varepsilon^{\prime} \gg \varepsilon \varepsilon^{\prime \prime}$, this gives the familiar result $n^{\prime} \approx\left(\varepsilon^{\prime}\right)^{\frac{1}{2}}$ and $n^{\prime \prime} \approx \varepsilon^{\prime \prime} / 2\left(\varepsilon^{\prime}\right)^{\frac{1}{2}}$. If the matrix medium is not a vacuum $\left(m^{*}=1\right)$, then the value of $m^{*}$ used in the equations should be $m_{\mathrm{SC}}^{*} / m_{\text {matrix }}^{*}$ where $m_{\mathrm{SC}}^{*}$ is the complex reflective index of the scatterer and $m_{\text {matrix }}^{*}$ is the complex reflective index of the medium. In the case of ice and water at $60 \mathrm{MHz}$ and close to the melting point $\left(\varepsilon^{*}{ }_{\mathrm{w}}=83-i 0.027\right.$ (Kaye and Laby, 1975) and $\left.\varepsilon^{*}{ }_{i}=3.17-i 0.014\right), m^{*}=5.12$ - i0.0088. $\Psi_{n}(z)$ and $\zeta_{n}(z)$ are known as the Riccati Bessel functions and are defined as

$$
\begin{aligned}
& \Psi_{n}(z)=z j_{n}(z) \\
& \zeta_{n}(z)=z h_{n}^{(2)}(z)
\end{aligned}
$$

where $j_{n}$ and $h_{n}^{(2)}$ are the spherical Bessel functions of the first and third kind (tabulated in, for example, Abramowitz and Stegun (1964)).

To obtain the $n$th Riccati function, it is possible to use the recurrence relations derived for the spherical functions

$$
f_{n+1}=\left[\frac{2 n+1}{z}\right] f_{n}-f_{n-1}
$$

and

$$
\frac{\mathrm{d}}{\mathrm{d} z} f_{n}=f_{n-1}-\frac{n+1}{z} f_{n}
$$

where $f_{n}=j_{n}$ or $h_{n}^{(2)}$. Equation (6) must be adapted so that it can be applied to the Riccati functions (for Equation (5) this is trivial) and doing this we obtain

$$
\frac{\mathrm{d} g_{n}}{\mathrm{~d} z}=g_{n-1}-n g_{n} / z
$$

where $g_{n}=\psi_{n}$ or $\zeta_{n}$.

Given the first two spherical Bessel functions, it is thus possible to calculate any number of Riccati functions from Equations (5) and (7), and hence the scattering amplitude, $S_{1}\left(180^{\circ}\right)$.

If the radar cross-section $\sigma$ is defined as $4 \pi$ times the power scattered back per steradian divided by the power incident on a unit area, then 


$$
\sigma=\frac{4 \pi}{k^{2}}\left|S_{1}\left(180^{\circ}\right)\right|^{2}=\frac{\lambda^{2}}{\pi}\left|S_{1}\left(180^{\circ}\right)\right|^{2}
$$

where $k=$ wave number (hence for an isotropic scatterer, $\sigma=$ the geometric cross-section $\left(\pi a^{2}\right)$ ).

Combining Equations (1) and (8) gives $P_{\mathrm{r}}$ for a single target. Assuming there are $f$ scatterers per $\mathrm{m}^{3}$ and that their volume fraction is, for example, 0.01 , then $f=$ $0.01 \times 3 /\left(4 \pi a^{3}\right)$. The volume illuminated is $2 \pi r^{2} l / G$, where $l$ is the pulse length in ice (in units of length) and the total scattered power at the receiver is then

$$
P_{\mathrm{r}} \text { (scat) }=\frac{P_{\mathrm{t}} G \lambda^{2} l}{32(\pi r)^{2}} f \sigma .
$$

The power-reflection coefficient (PRC) $R$ of a plane surface may be defined as

$$
P_{\mathrm{r}}=P_{\mathrm{t}} R\left[\frac{G \lambda}{8 \pi r}\right]^{2}
$$

and thus the effective PRC due to layer scattering is

$$
R_{\mathrm{sca}}=\frac{2 l}{G} f \sigma .
$$

\section{RESULTS AND DISCUSSION}

In Figure $1, R_{\mathrm{sca}}$ is plotted against the target radius $a$ for a volume fraction of 0.01 for three different scattering
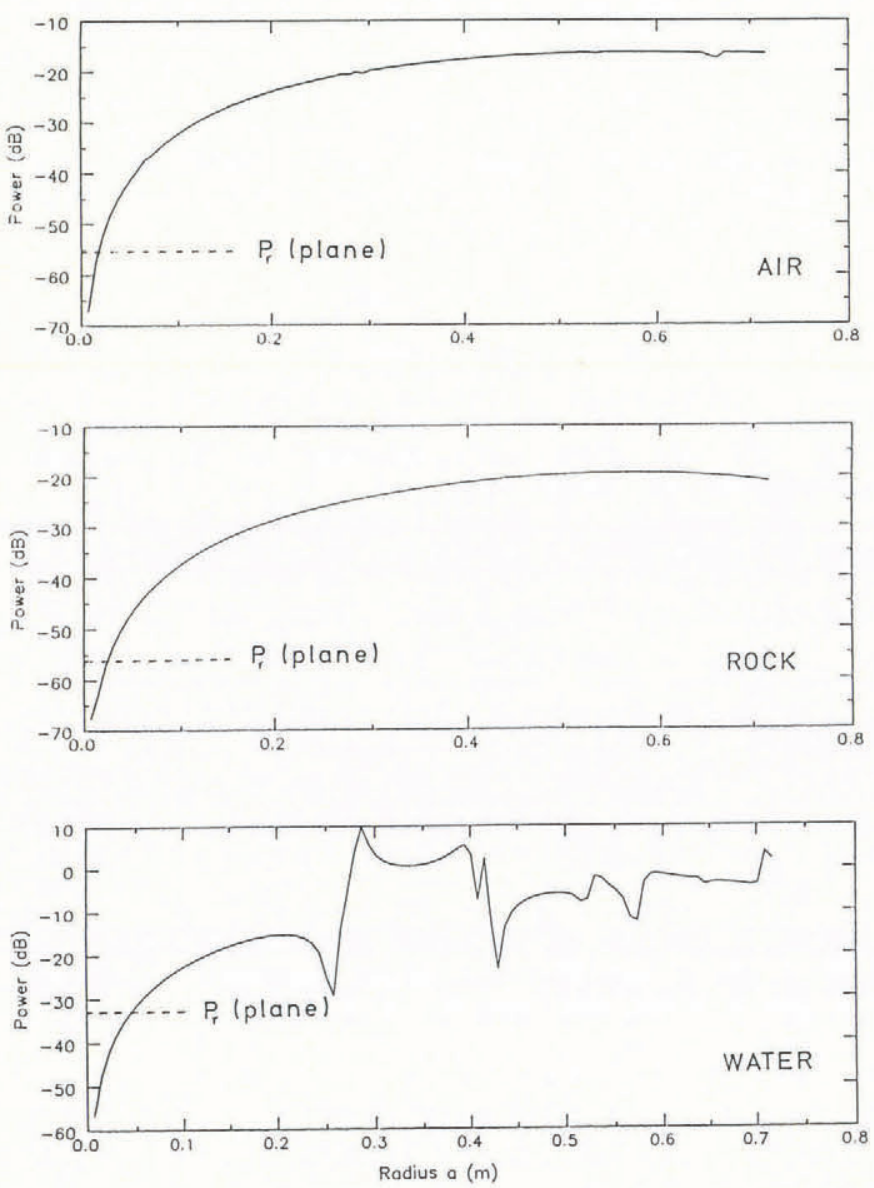

Fig. 1. The returned power $P_{r}$ in $d B$ (relative to a perfectly reflecting plane boundary) for a layer of spherical inclusions in ice plotted against their radii. The relative permittivity of the rock was taken as 5.0 and its conductivity as $1 \mu \mathrm{S} \mathrm{m}^{-1}$. For water, the former was 83 and the latter $90 \mu \mathrm{S} \mathrm{m}^{-1}$. The volume fraction, for all three types of inclusion, was 0.01 . materials. In (a) the inhomogeneity is air, in (b) it is rock $\left(\varepsilon^{*}=5-i 0.003\right)$, and in (c) it is water $\left(\varepsilon^{*}=\right.$ $83-i 0.027)$. It should be noted that the complex refractive index is frequency-dependent and that the data are only applicable for a value of $60 \mathrm{MHz}$. Marked on the $y$-axes are the PRCs calculated using Rayleigh's formula for a heterogeneous mixture of two dielectrics (van Beek, 1967) combined with Fresnel's equation for reflections at a plane dielectric boundary. It can be seen that $R_{\mathrm{sca}}>R_{\text {plane }}$ occurs for water when $a>25 \mathrm{~mm}$, for rock when $a>30 \mathrm{~mm}$, and for air when $a>20 \mathrm{~mm}$. Although water or air inclusions of these dimensions are probably not common for cold ice, in temperate glaciers they are more likely to exist and have been observed in a number of studies (e.g. Hodge, 1976; Schommer, 1977).

The positive reflection coefficients in Figure 1 require some explanation. They occur for several reasons, the most important of which is that the source and receiver have point geometry. It should also be noted that power is being returned from a volume and not a surface, and that it is possible for $\sigma$ to be greater than one (i.e. the effective area is greater than its geometrical area). Figure 2 is a schematic

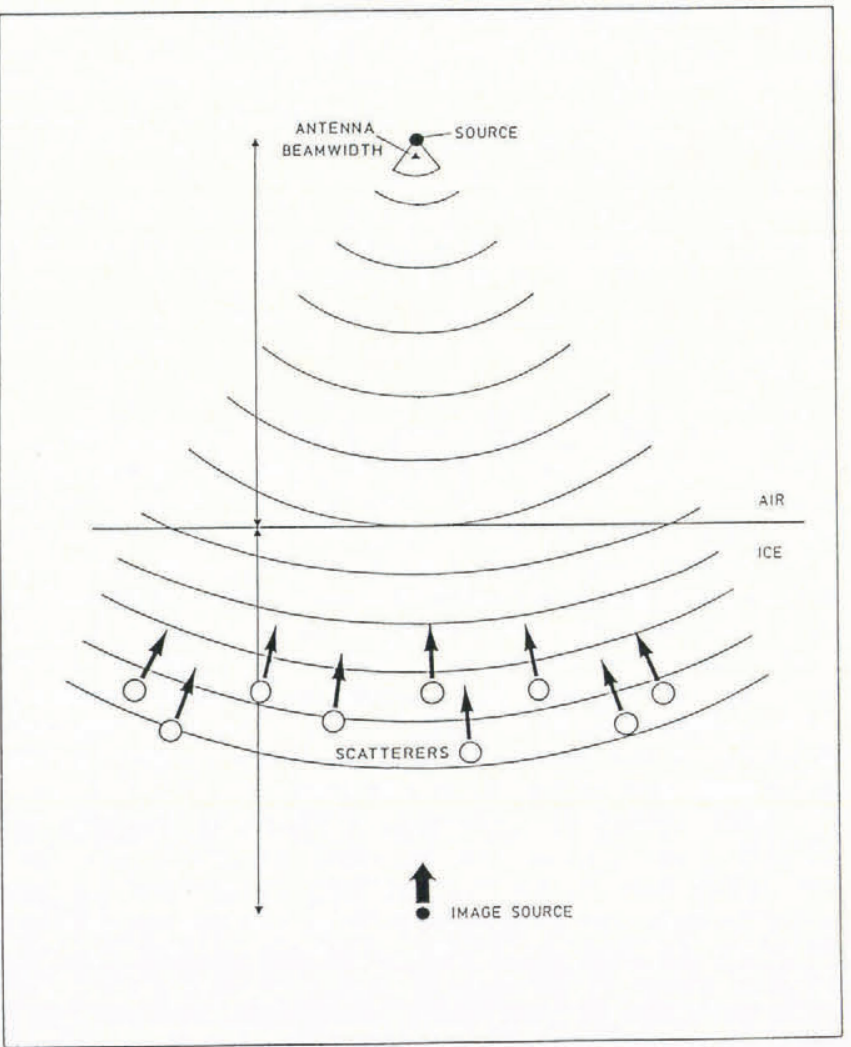

Fig. 2. Schematic diagram of scattering from a layer of spherical inclusions in ice compared with the back-scattered power from a perfectly reflecting plane boundary. Using the method of images, it can be shown that the latter is equivalent to placing an identical source (the image source) at the same distance from the plane as the original source but on the other side.

diagram illustrating how a layer of individual scattering centres can return more power to the source than a perfectly reflecting plane boundary (i.e. a surface with a PRC of 1). In the latter case, $P_{r}$ is equivalent to that of a source, strength $P_{\mathrm{t}}$, positioned $2 \times r$ from the antenna. For spherical scatterers, however, power is returned from a broad swath primarily determined by the antenna beam width. Combined with the large back-scattering cross-section that water bodies may possess (Fig. 3), the returned power can exceed that of a perfectly reflecting plane boundary. This does not violate conservation of energy because the power returned to the receiver from the plane comes from only one point on that plane directly below the source.

In Figure $3, \sigma / \pi a^{2}$ is plotted against $x$ and from this we observe the interesting result that, for certain values of 

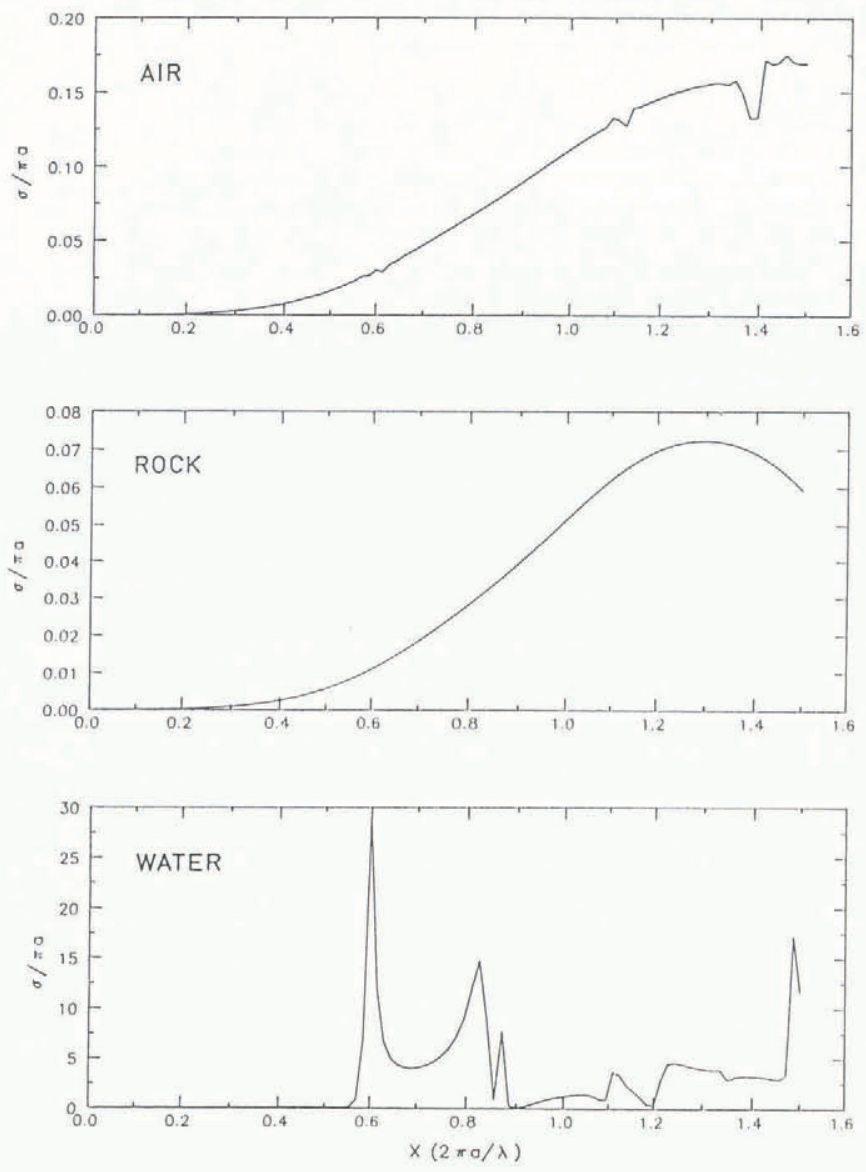

Fig. 3. Back-scattering cross-sections for air, rock, and water in ice plotted against the dimensionless parameter $X$. Values of the constants are as for Figure 1.

$a, \sigma>4 \pi a^{2}$ (i.e. the effective area is greater than that of a sphere). The remarkable scattering diagram obtained for water is a consequence of the large value of $\varepsilon^{\prime}$ and its finite conductivity. These combine to produce a resonant component to the back-scattered power. Such a phenomenon is well known in optical physics (Conwell and others, 1984; Probert-Jones, 1984) but has not, to the author's knowledge, previously been considered in the case of radar sounding. In light-scattering studies, the permittivities are of ten assumed to be real (i.e. the dielectric media are non-conducting) but, given a large enough discontinuity in relative permittivity, resonsant effects may still be observed. The conductivity used in the calculations was $9 \times 10^{-5} \mathrm{~S} \mathrm{~m}^{-1}$ (Wolff and Paren, 1984). Varying this value within a reasonable range can reduce the cross-section by up to a factor of 2 but represents a reduction in the PRC of only $3 \mathrm{~dB}$.

\section{Internal reflecting horizons in Spitsbergen}

On a number of glaciers in Spitsbergen, radio echosounding records have indicated the existence of a single internal reflecting horizon (IRH) (Bamber, 1987) with PRCs in the range -15 to $-30 \mathrm{~dB}$. These values are considerably higher than previously recorded values for an internal reflector (e.g. Ackley and Keliher, 1979; Millar, unpublished) and could be explained if the reflection were due to water bodies on the decimetre scale. Such an hypothesis is supported by the results of Soviet studies on a number of Spitsbergen glaciers using a higher frequency (and hence shorter wavelength) of $440 \mathrm{MHz}$ (personal communication from Y.Y. Macharet). The Soviet workers found that some of the IRHs appeared on their RES records as a number of individual overlapping hyperbolae lying at a fairly uniform depth and forming a surface of reflectors.

It has been shown that the level of the IRH is well described by the piezometric surface of a Röthlisberger channel (Bamber, 1987). It is suggested, therefore, that the
IRHs (which were observed in the spring, before the melt season had started) are remnants of the large-scale network of conduits which drain the surface melt water to one or more Röthlisberger channels. Some of these conduits are closed off in the winter, trapping water in cavities which are too deep to be influenced by the cold winter wave so that the ice below this depth remains at, or very close to, its melting point. It is likely, however, that some of the cavities are air-filled, much or all of the water having successfully drained away.

In conclusion, it can be stated that when the target radius is greater than about $50 \mathrm{~mm}$ the scattering power (for the dispersoids considered) will outweigh that which would be expected from a plane dielectric boundary which is assumed to obey a relaxation process due to interfacial polarization (van Beek, 1967). For the case of water bodies, if $a$ is greater than $\approx 25 \mathrm{~mm}$, the scattered power can be greater than that for any plane boundary. This result clearly illustrates the difficulties that may be encountered when sounding temperate glaciers possessing englacial water bodies. It could explain the problems that were found in obtaining bed echoes during the melt season in Svalbard by Soviet workers (Dowdeswell and others, 1984) and the fact that bed returns are of ten absent in the accumulation zone of a glacier. It might also explain some of the results of Davis (unpublished), who found considerable internal scattering on temperate glaciers in the European Alps. He deduced, by an empirical approach, a mean attenuation of the wave of up to $10 \mathrm{~dB} / 100 \mathrm{~m}$ and associated this with a combination of the effects of scattering and dielectric absorption. This work strongly supports the suggestion that the most suitable sounding frequencies for temperate glaciers are the lowest ones (e.g. Watts and England, 1976).

\section{ACKNOWLEDGEMENTS}

The author would like to thank $\mathrm{Dr}$ S. Evans and Professor G.K.C. Clarke for their advice and comments. Funding was through a U.K. Natural Environment Research Council grant GR3/4463 and a NERC studentship to the author.

\section{REFERENCES}

Abramowitz, M. and I.A. Stegun. 1965. Handbook of mathematical functions. New York, Dover Publications.

Ackley, S.F. and T.E. Keliher. 1979. Ice sheet internal radio-echo reflections and associated physical property changes with depth. J. Geophys. Res., 84(B10), 5675-5680.

Bamber, J.L. 1987. Internal reflecting horizons in Spitsbergen glaciers. Ann. Glaciol., 9, 5-10.

Bamber, J.L. Unpublished. Radio echo sounding studies of Svalbard glaciers. (Ph.D. thesis, University of Cambridge, 1987.)

Beek, L.K.H. van. 1967. Dielectric behaviour of heterogeneous systems. Prog. Dielectr., 7, 69-114.

Conwell, P.R., P.W. Barber, and C.K. Rushworth. 1984. Resonant spectra of dielectric spheres. J. Opt. Soc. Am., I(1), 62-67.

Davis, J.L. Unpublished. The problem of depth sounding temperate glaciers. (M.Sc. thesis, University of Cambridge, 1973.)

Dowdeswell, J.A., D.J. Drewry, O. Liestøl, and O. Orheim. 1984. Radio echo-sounding of Spitsbergen glaciers: problems in the interpretation of layer and bottom returns. J. Glaciol., 30(104), 16-21.

Hodge, S.M. 1976. Direct measurement of basal water pressures: a pilot study. J. Glaciol., 16(74), 205-218.

Hulst, H.C. van de. 1957. Light scattering by small particles. New York, Dover Publications.

Kaye, G.W.C. and T.H. Laby. 1975. Tables of physical and chemical constants. 14th edition. London, Longman Press.

Millar, D.H.M. Unpublished. Radio echo layering in polar ice sheets. (Ph.D. thesis, University of Cambridge, 1981.) 
Probert-Jones, J.R. 1984. Resonance component of backscattering by large dielectric spheres. J. Opt. Soc. Am., 1(8), 822-830.

Schommer, P. 1977. Wasserspiegelmessungen im Firn des Ewigschneefeldes (Schweizer Alpen) 1976. Z. Gletscherkd. Glazialgeol., 12(2), 125-141.
Watts, R.D. and A.W. England. 1976. Radio-echo sounding of temperate glaciers: ice properties and sounder design criteria. J. Glaciol., 17(75), 39-48.

Wolff, E.W. and J.G. Paren. 1984. A two-phase model of electrical conduction in polar ice sheets. J. Geophys. Res., 89(B11), 9433-9438.

MS. received 23 April 1988 and in revised form 26 July 1988 